\title{
Control of SIMO Systems in Simulation: The Challenge of the Multiple Axes Actuating Pneumatic Arm
}

\author{
G. P. Smyrnaiou \\ Dept. of Automation \\ Engineering \\ PUAS, Athens, \\ Greece
}

\author{
M. Papoutsidakis \\ Dept. of Automation \\ Engineering \\ PUAS, Athens, \\ Greece
}

\author{
A. Xatzopoulos \\ Dept. of Automation \\ Engineering \\ PUAS, Athens, \\ Greece
}

\author{
D. Tseles \\ Dept. of Automation \\ Engineering \\ PUAS, Athens, \\ Greece
}

\begin{abstract}
In this paper a comparative study of the classical control methods for the testing of a mathematical model, which controls six actuators of a six degrees of freedom robotic arm with a single controller, is illustrated, aiming to the constructive simplification of the system. In more detail, a mathematical model of the system is designed which simulates all mechanical parts, including 5-way directional pneumatic valve, the pneumatic actuators/pistons and the mathematical model of the controller. The purpose of the above is the tuning of a Single Input, Multiple Outputs (SIMO) controller which will direct the motion of the six pneumatic pistons. The thorough analysis of the implementation of the pneumatic system in Matlab/Simulink environment is followed by experimentation and results using Proportional (P), Proportional-Integral (PI), ProportionalDerivative (PD) and Proportional-Integral-Derivative (PID) controllers. The simulation results show the advantages of the above classical control methods on the robotic human arm which imitating human motion and made by a well-known company in the field of pneumatic automation.
\end{abstract}

\section{Keywords}

Single input-multiple output systems, pneumatic positioning systems, simulation environment, PID controller design.

\section{INTRODUCTION}

In modern industry pneumatic systems (PS) are used with an increased rate. A pneumatic system uses compressed air as a mean to transfer the motion, and have as a final result the movement of a piston or in rare cases the rotation of a motor. Pneumatic Systems find application in cases where small forces should be applied and great velocity is needed. This is the main reason they are widely used in the industry. Pneumatic systems also store and transfer energy with ease and can produce low cost, linear movement with relatively high speed, easily adjustable [1].

Cylinders are considered the basic elements of motions in Pneumatic Systems, which convert the pneumatic force into a linear motion.

Pneumatic cylinders consist of a chamber and ports from where the air enters and exits, performing, that way, the linear movement. The piston's chamber is tight to avoid any leakage and is highly durable so that it can endure the high pressure [2].

There are three distinguished categories for the pneumatic cylinders:

- In the single-acting cylinders, which are divided into single-acting cylinders with and without rebound spring.
- In the double-acting cylinders, which are divided into double acting cylinders with a single rod, with doublerod, telescopic and with delay.

- In the Tandem cylinder, which includes two separated double-acting cylinders of compressing air, in a row for additional power. This setup doubles the air pressure provided by the piston because of its increased [3], [4].

For the correct function of the pneumatic actuators a controller is required. The valves are devices that help regulate the starting or stopping of the piston, and also assist in determining the compressed air flow direction. The valve controls the motion of the rod and the valve respectively is tuned by a classic controller [4].

Valves are distinguished in the following categories:

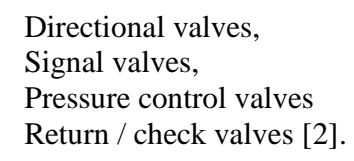

The requirements of modern times and particularly the last five years have imposed a rapidly developing technology in the hydraulic automation area. As a result, a new technology sector appeared: hydraulic. This remarkable growth is mainly due to the contribution of the construction of hydraulic components and P.L.C, general.

By hydraulic system we mean that a device used to transmit motion and power transmission from the motor to the driven machine. The analysis, design and modeling hydraulic automation systems, based on principles and laws, is widely applicable in fields such as the automotive industry and in robotics.

\section{CONTROL DESIGN IN SIMULATION ENVIRONMENT}

\subsection{Non-Linear Mathematical Model}

A pneumatic system consists of a cylinder, a valve, exhaust and supply tubes, position sensors and pressure sensors. The load is the rest of the mechanical parts that are connected to the pneumatic piston. A non-linear pneumatic system is illustrated in the following Figure. 


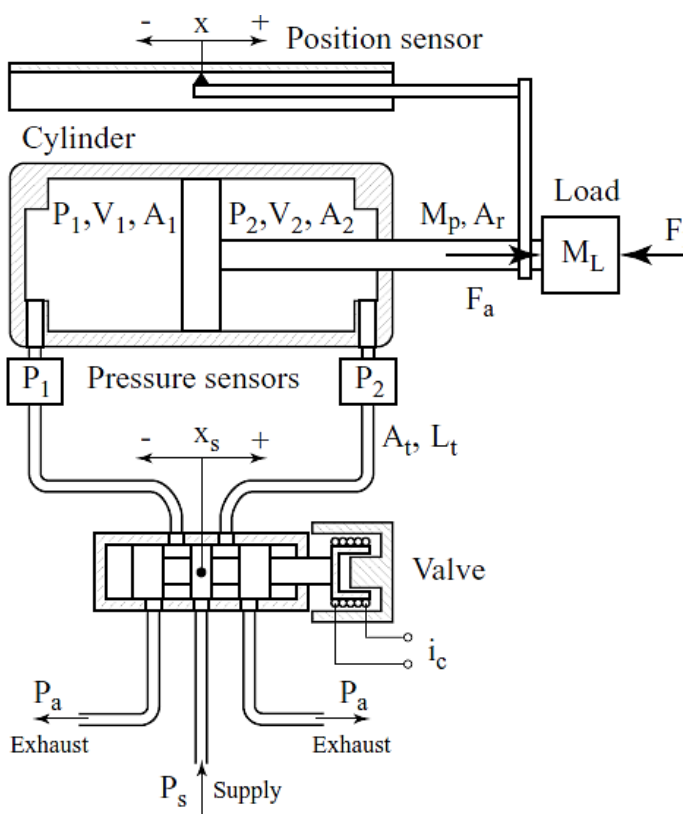

Fig. 1: Standard pneumatic cylinder-valve model

The equation of motion for the piston-rod-load assembly can be expressed as:

$$
\begin{aligned}
\left(\mathbf{M}_{\mathbf{L}}+\mathbf{M p}\right) \cdot \ddot{\mathbf{x}} & +\mathbf{B} \cdot \dot{\mathbf{x}}+\mathbf{F}_{\mathrm{f}}+\mathbf{F}_{\mathbf{L}} \\
& =\mathbf{P 1} \cdot \mathbf{A} 1-\mathbf{P} \mathbf{2} \cdot \mathbf{A} \mathbf{2}-\mathbf{P}_{\mathrm{a}} \cdot \mathbf{A}_{\mathbf{r}}
\end{aligned}
$$

Where $M_{L}$ is the external mass of the load, Mp is the mass of the piston, $x$ is the current position of the rod, $\dot{x}$ is the speed of the piston, $\ddot{\mathrm{X}}$ is the acceleration of the piston, $\mathrm{B}$ is the friction coefficient, $F_{f}$ is the friction force (Coulomb), $F_{L}$ is the external force, $\mathrm{P} 1$ and $\mathrm{P} 2$ are the absolute value of the air pressure of the actuator's chamber, $\mathrm{P}_{\mathrm{a}}$ is the absolute value of the environment pressure, $\mathrm{A} 1$ and $\mathrm{A} 2$ are the piston effective areas, $A_{r}$ is the rod cross sectional area.

The above equation represents the force of the actuator generated by different pressures acting on the opposite sides of the piston, in order to control the actuator's output.

The time derivative for the pressure in the pneumatic cylinder chambers can be expressed as:

$$
\begin{array}{r}
\dot{P}_{i}=\frac{R T}{V_{0 i}+A_{i}\left(\frac{1}{2} L \pm x\right)}\left(a_{\text {in }} \dot{\mathbf{m}}_{\text {in }}-a_{\text {out }} \dot{\mathbf{m}}_{\text {out }}\right) \\
-\mathbf{a} \frac{P_{i}}{V_{0 i}+A_{i}\left(\frac{1}{2} L \pm x\right)} \dot{\mathbf{x}}
\end{array}
$$

Where $\mathrm{P}$ is the piston's pressure, $\mathrm{R}$ is the ideal gas constant, $\mathrm{T}$ is the temperature, $\mathrm{V}_{0 \mathrm{i}}$ is the inactive volume at the end of stroke and admission ports, $A_{i}$ is the piston effective area, $L$ is the piston stroke, $\mathrm{x}$ is the piston position, $\mathrm{m}_{\text {in }}$ and $\mathrm{m}_{\text {out }}$ are the mass flows entering and leaving the chamber, and $a_{\text {in }}$ and $\mathrm{a}_{\text {out }}$ taking values between 1 and $\mathrm{k}$, depending on the actual heat transfer during the process.

The first term of the above equation represents the effect of the pressure of the air flow into or out of the cylinder chamber, while the second term describes the effect of the motion of the piston.

The pressure drop across the valve orifice is usually large, and the flow has to be treated as compressible and turbulent. If the upstream to downstream pressure ratio is larger than a critical value $\mathrm{P}_{\mathrm{cr}}$, the flow will attain sonic velocity (choked flow) and will depend linearly on the upstream pressure. If the pressure ratio is smaller than $\mathrm{P}_{\mathrm{cr}}$ the mass flow depends nonlinearly on both pressures.

The standard equation for the mass flow through an orifice of area $A_{u}$ is:

$$
\dot{m}_{u}= \begin{cases}C_{f} A_{u} C 1 \frac{P_{u}}{\sqrt{T}} & \text { if } \frac{P_{d}}{P_{u}} \leq P_{c r} \\ C_{f} A_{u} C 2 \frac{P_{u}}{\sqrt{T}}\left(\frac{P_{d}}{P_{u}}\right)^{\frac{1}{k}} \sqrt{1-\left(\frac{P_{d}}{P_{u}}\right)^{\frac{(k-1)}{k}}} & \text { if } \frac{P_{d}}{P_{u}}>P_{c r}\end{cases}
$$

Where $\dot{\mathrm{m}}_{\mathrm{u}}$ is the mass flow through valve orifice, $\mathrm{C}_{\mathrm{f}}$ is the supply rate, $P_{u}$ is the value of the upstream pressure and $P_{d}$ is the value of the downstream pressure. $\mathrm{C} 1$ and $\mathrm{C} 2$ are constants of a given fluid, in this case the air:

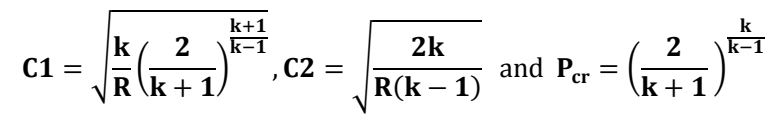

Where $\mathrm{k}$ is the constant of air value [5],[6].

\subsection{Simulation of the Pneumatic System with 6 Actuators and P Controller}

The pneumatics' system simulation model with six parallel connected pistons with analog control is illustrated in below Figure 2:

This model represents an integrated pneumatic system with six controllable actuators/ pistons. The components are described below:

\section{- A directional control valve 5/3}

This is a five-way valve and three-position ( $5 / 3$ valve) which is used to control the compressed air's flow into and out thepiston, having as an output the return of the piston. This valve has an intermediate position, which is closed, the transits B-X and A-S stay closed. That way the compressed air gets trapped in the champers of the piston. The plunger balances under the influence of the two pressures, and stop in an intermediate position, with a relative accuracy. The plunger is immobilized that way and the rod remains stable. As shown in Fig.2, the supplied pressure is inserted in port $\mathrm{P}$ and the pneumatic actuator is connected to ports $\mathrm{A}$ and $\mathrm{B}$. $\mathrm{R}$ and $\mathrm{S}$ are return paths of the $\mathrm{A}$ and $\mathrm{B}$ ports respectively.

\section{- Double-acting Cylinder}

The Pneumatic System uses a double-acting cylinder with a single rod that forces the compressed air to move the rod in both directions.

\section{- $\quad$ Flow Rate}

As shown from Fig. 2, the block diagram of the flow rate of the pneumatic system is connected with a directional valve to drive the compressed air to the actuators ports so that he can move accordingly. The Flow rate block diagram contains a subsystem which consists:

- Mass \& Heat Flow Sensor, which is a device that converts the mass flow rate and heat flow rate between the two pneumatic nodes into physical measurement signals $\mathrm{G}$ and $\mathrm{Q}$. The positive direction of the sensor is from the port A to port B.

- PS-Simulink Converter which converts the input Physical Signal to a unit less Simulink output signal in this case $\mathrm{Kg} / \mathrm{s}$. 


\section{- Pressure Source}

This is an ideal air compressor which maintains a fixed pressure differential, irrespectively the flow rate. The compressor does not add extra heat to the system. A positive pressure difference results in the pressure in port $\mathrm{B}$ to be greater than the pressure in the port $\mathrm{A}$. The compressor differential pressure is 6 bar.

\section{- Subsystem block and Time scope block}

For the pneumatic system Simulation, the Simulink library was used. Time scope block is used and the six actuators are connected and also the input step. A Subsystem block is created which includes the 5 pneumatic pistons and it's connected with the first one (Master-slaves) as illustrated in the following figure.

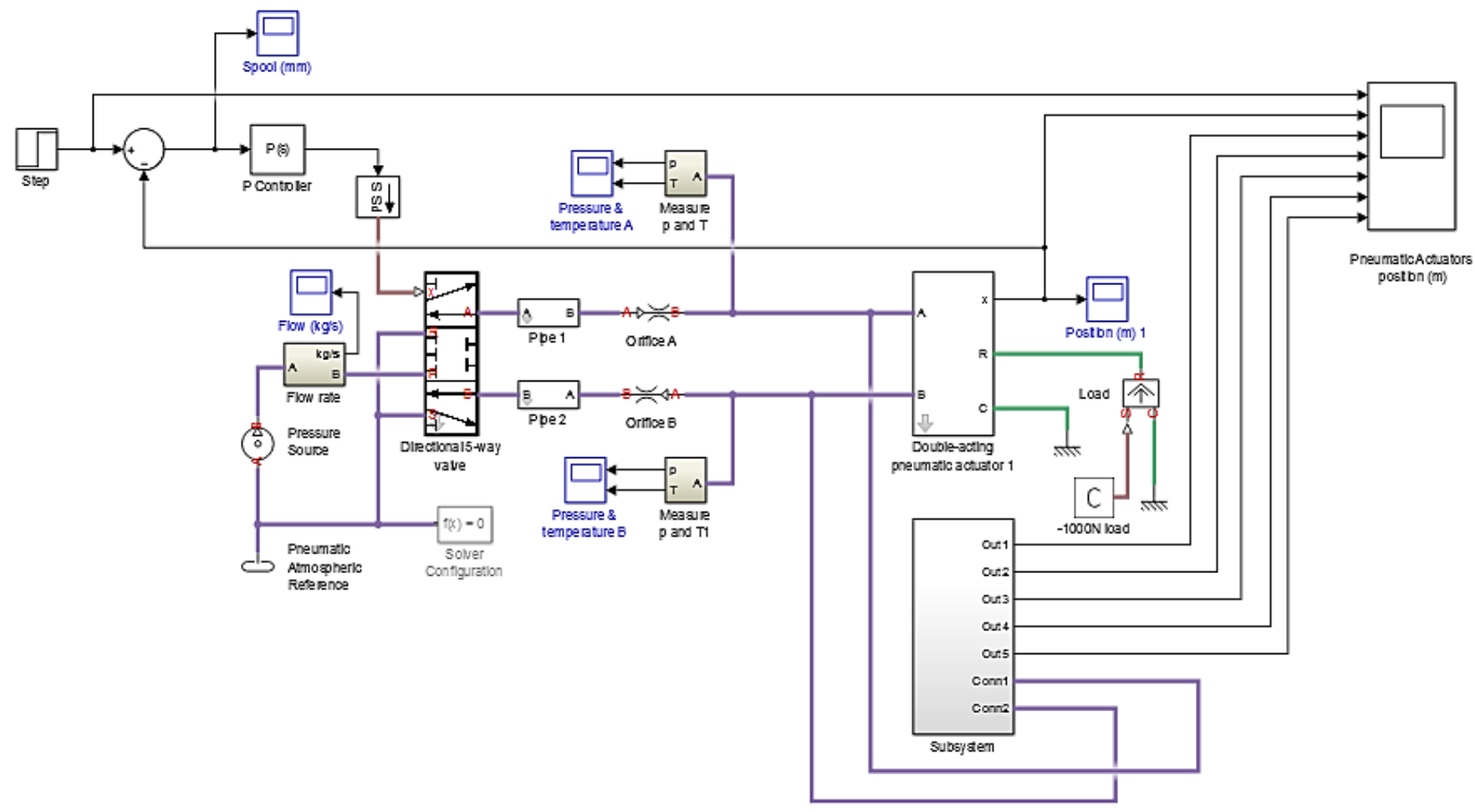

Fig 2: Simulation Model of the Overall System

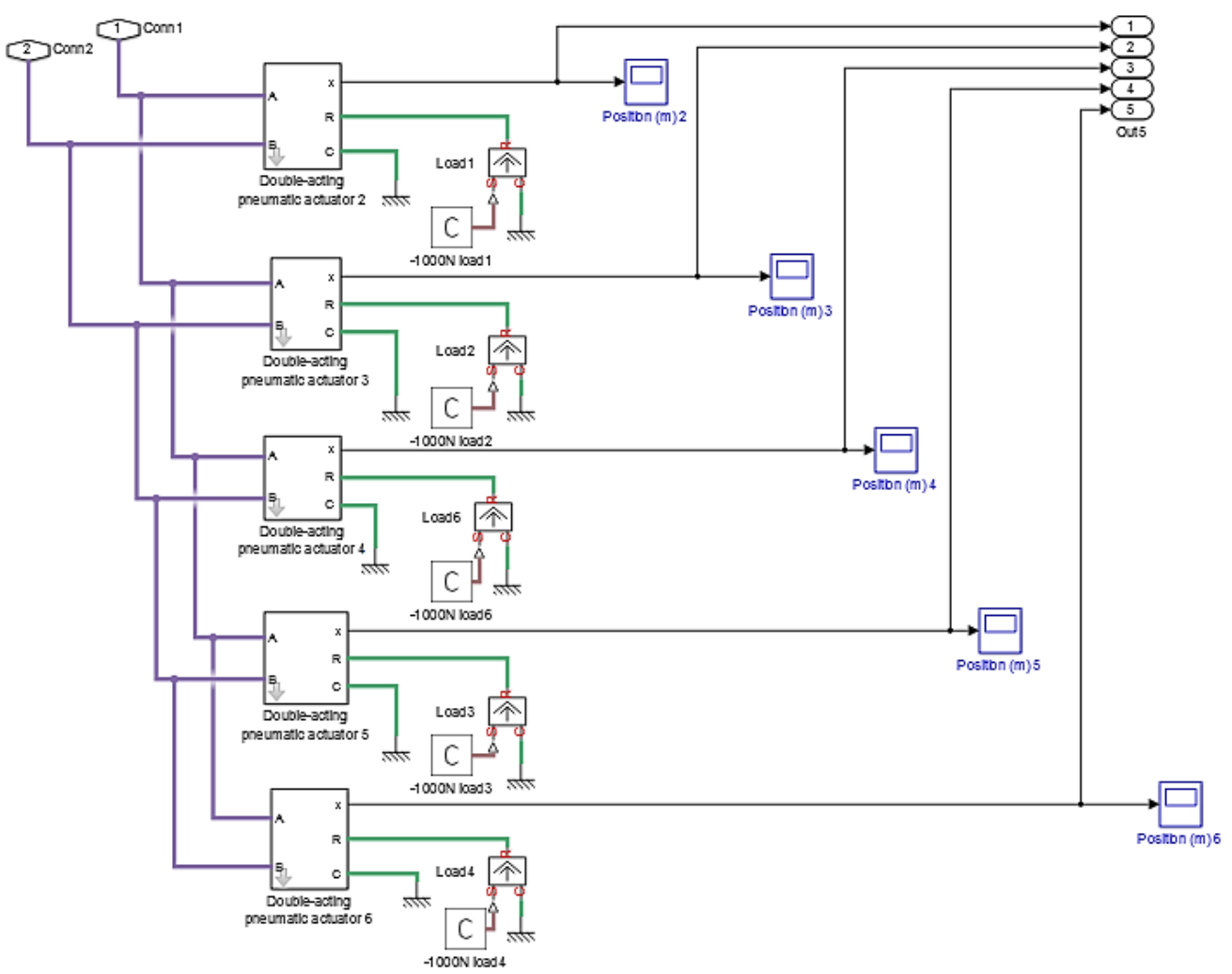

Fig 3: Subsystem Block 
The Step input is set to start from 0 sec. Step's range value starts from 0 to 1 . As final value in the stoke parameter of the piston is set $0.05 \mathrm{~m}$ height and the stroke length is set in 0.1 $\mathrm{m}$. From the Simulink library a PID controller is selected (Proportional-Integral-Derivative) the $\mathrm{P}$ value is set to $K_{P}=120$ and the rest of the parameters are eliminated (0) to achieve Proportional control. Simulation time is set to $\mathrm{T}=0.5$ sec, air supply pressure is set to 6 bar, $\left[6 \cdot 10^{5}\right.$ Pascal $]$. The systems response and the position of the six actuators with Proportional control are shown in the following figure.

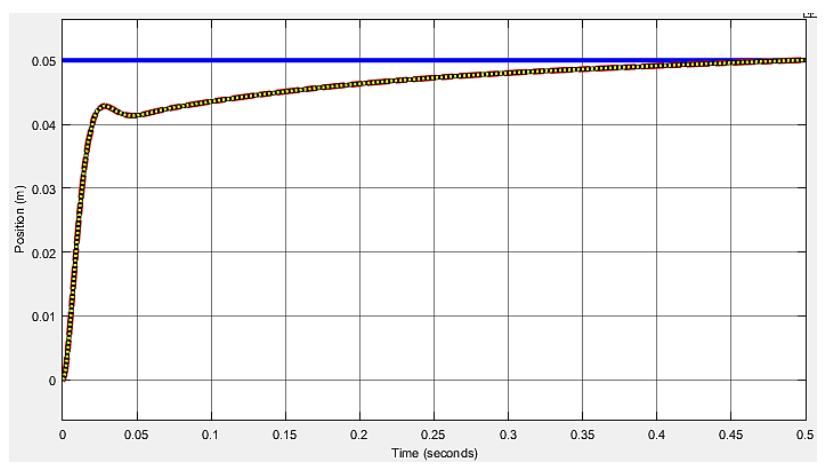

Fig 4: $P$ controller's Step Response

The fig. 4 results for $K_{P}=120$, where the pistons have settled in the Set value. For lower $K_{P}$ values a steady state error occurs in the systems response and for greater $K_{P}$ values the steady state error is lifted. At this point it should be mentioned, that the steady state error is not erased completely, but instead it is getting smaller.

The Step input is set to start from $0 \mathrm{sec}$. As final value in the stoke parameter of the piston is set $0.05 \mathrm{~m}$ height and the stroke length is set in $0.1 \mathrm{~m}$. The PID controller is tuned (Proportional-Integral-Derivative) to $K_{P}=120$ and $K_{I}=5$ to achieve Proportional-integral control. Simulation time is set to $\mathrm{T}=0.5 \mathrm{sec}$, air supply pressure is set to $6 \mathrm{bar},\left[6 \cdot 10^{5}\right.$ Pascal]. The following figure illustrated the pneumatic system response.

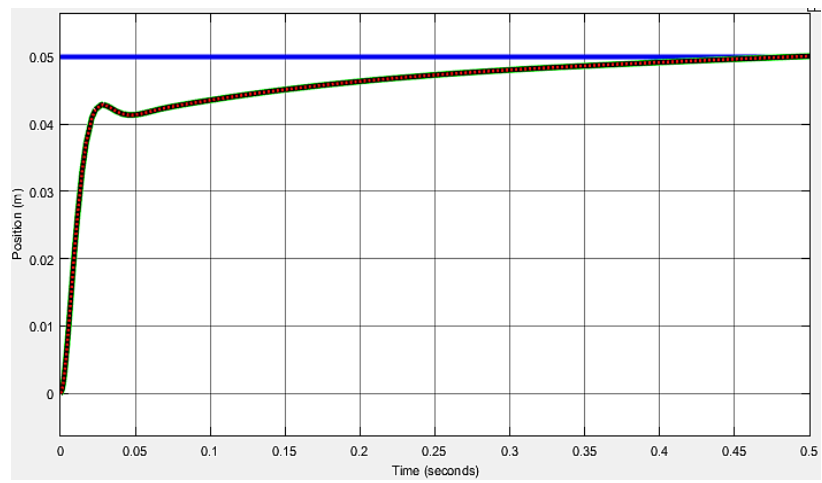

Fig 5: PI controller's Step Response

The above figure's results for $K_{P}=120$ and $K_{I}=5$, where the pistons have settled in the Set value. For increased the $\mathrm{K}_{\mathrm{I}}$ gain, a steady state error appears in the system with values of the position of the pistons greater than $0.05 \mathrm{~m}$.

The Step input is set to start from 0 sec. As final value in the stoke parameter of the piston is set $0.05 \mathrm{~m}$ height and the stroke length is set in $0.1 \mathrm{~m}$. The PID controller is tuned (Proportional-Integral-Derivative) to $\mathrm{K}_{\mathrm{P}}=120$ and $\mathrm{K}_{\mathrm{D}}=$ 0.6 to achieve Proportional-Derivative control in the pneumatic system. The systems response and the position of the six actuators with Proportional-Derivative control is shown in the following figure. Simulation time is set to $\mathrm{T}=0.5$ sec, air supply pressure is set to $6 \mathrm{bar},\left[6 \cdot 10^{5} \mathrm{Pascal}\right]$.

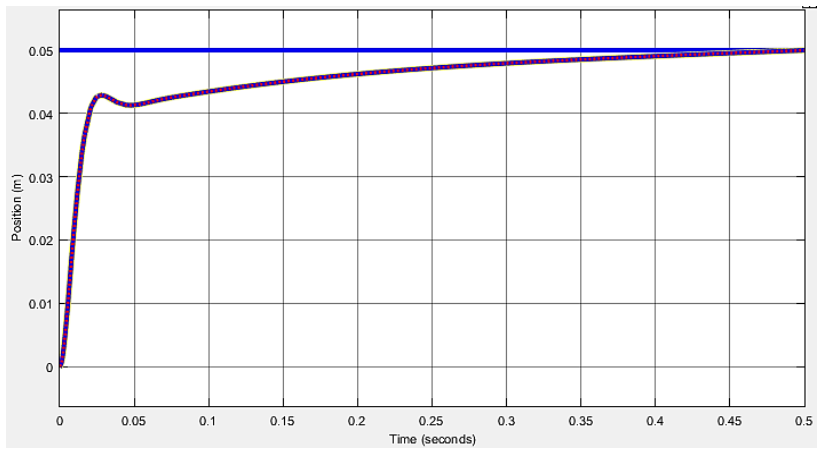

Fig 6: PD controller's Step Response

The above figure, results for $K_{D}=0.6$ and $K_{P}=120$ and filter coefficient $\mathrm{N}=100$, where the pistons have settled in the Set value. Increasing the $K_{D}$ coefficient a steady state error occurs with values on the position of the pistons less than 0.05 $\mathrm{m}$.

The Step input is set to start from $0 \mathrm{sec}$. As final value in the stoke parameter of the piston is set $0.05 \mathrm{~m}$ height and the stroke length is set in $0.1 \mathrm{~m}$. The PID controller is tuned (Proportional-Integral-Derivative) to $\mathrm{K}_{\mathrm{P}}=120, \mathrm{~K}_{\mathrm{I}}=5$ and $\mathrm{K}_{\mathrm{D}}=0.6$ to achieve Proportional-Integral-Derivative control in the pneumatic system. The systems response and the position of the six actuators with Proportional-IntegralDerivative control is shown in the following figure. Simulation time is set to $T=0.5 \mathrm{sec}$, air supply pressure is set to 6 bar, $\left[6 \cdot 10^{5}\right.$ Pascal $]$.

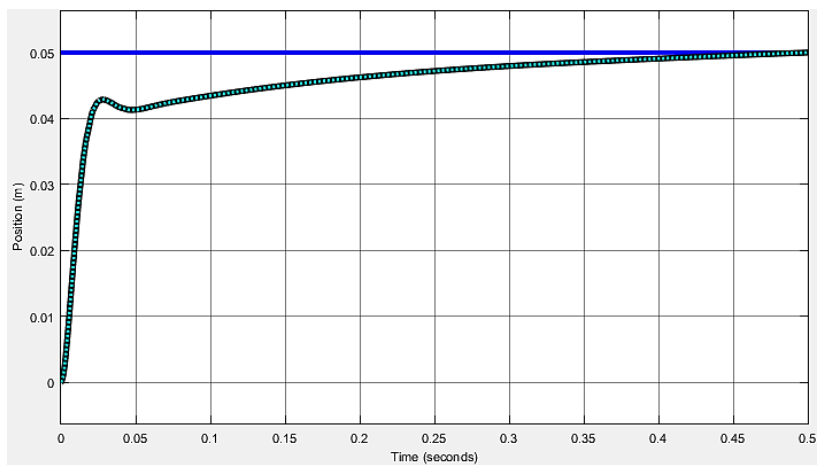

Fig 7: PID controller's Step Response

The above figure results for $\mathrm{K}_{\mathrm{P}}=120, \mathrm{~K}_{\mathrm{I}}=5 \mathrm{~K}_{\mathrm{D}}=0.6$ and filter coefficient $\mathrm{N}=100$, the pistons have settled in the Set value. Increasing the gains values changes occur in the steady state error. Also distortion may appear on some situations for increased gain values. Increasing the Proportional gain while leaving the other two gains aside it is observed, that the system's response tries to follow the input set to it but a steady state error occurs. Increasing its value the steady state error is getting smaller but it is never eliminated. Increasing the Integral gain and leaving the other coefficients intact the steady state error is getting smaller but we have distortion. By increasing the Derivative gain the overshot is getting reduced, as well as the settling time while the steady state error get smaller changes.

With the Proportional control method the Pneumatic system response gets accurate by choosing the correct value and acts fast with settling time $\mathrm{Ts}=492.4 \mathrm{~ms}$ (Fig.4). When the application requirements are increased then this method of 
control gets unsuitable and the systems response with a load gets unpredictable [7].

Therefore this method of control cannot deal with all the scenarios that can occur in the industrial environment and needs furthermore assistance from a subsystem. The second method of control that was used in the pneumatic system was the Proportional-Integral method which according to the theory using the integration term is intended to eliminate the steady state error in the system. The steady state error theoretically should have been eliminated by the integral term while adding some minor distortion on the systems response, has settling time Ts=488.6 ms doesn't eliminate the stead state error Ess $=0.05 \mathrm{~m}$ as expected even at the maximum value (Fig 5).

In simulation environment the PI controller doesn't add oscillating behavior, nor creates overshoot in the pneumatic system. In reality studies have shown that the Integral coefficient on a Non-linear system adds constantly force in the system creating disturbances [7],[8],[9],[10], and unbalancing the systems response. That is the main reason that the PI controller is avoided in general in both simulation experiments as well as in real once.

The next method of control that was applied in the pneumatic system was the PD controller (Proportional-Derivative). TheD coefficient should minimize the systems overshoot. With experimental testing it is confirmed that the PD controller helps stabilize the Pneumatic system response with Ts=498.7 $\mathrm{ms}$ and eliminates the over shoot with a Ess=0.05 (Fig.6).

The last controller tested was the three-term PID controller (Proportional-Integral-Derivative) to complete the comparison between all the controllers. The response time of the PID controller it's the same as the PD's. Ts=498.5 ms (Fig.7). In simulation environment, PID controller has distortion when the coefficients value are too high but he is eliminating the overshoot effect.

In reality the presents of the I-term is creating low frequency signals that are hard to eliminate and adds unpredictable behavior in the system's response [7],[8],[9],[10], making it hard to justify its usage on a non-linear model as this one.

Therefore the most optimal response for the six actuator pneumatic systems is given by PD controller in Simulation as well as in real experiments.

\section{CONCLUSIONS AND DISCUSSION}

In the current study a comparison was performed between classic methods of control in simulation environment that included a six actuator robotic arm with six degrees of freedom and a single classic controller. The design and implementation of the pneumatic system was described (Matlab/Simulink). The mathematical models of the directional valve, the actuators and the controllers were described. The experimental result where Proportional (P), Proportional-Integral (PI), Proportional Derivative (PD), and Proportional-Integral-Derivative (PID) were used, were illustrated. The innovative pneumatic system simulation model of an input and multiple output (Single Input, Multiple Outputs - SIMO), gives the user the ability to tune in a single controller that can simultaneously drives six parallel connected drive pistons. After the experiments completion it was shown that the Proportional Derivative (PD) provided the system with the most optimal response.

Moreover, the specific pneumatic system with the given robustness and repeatability, as recorded by the experimental procedure, created a clear practical promises for the usage of the robotic hand ExoHand of Festo which can be used as an outer skeleton that can be worn as a glove by the human hand.

The fingers can be actively moved and their strength amplified the operator's hand movements are registered and transmitted to the robotic hand in real time. The exoskeleton hand has all the principal physiological degrees of freedom of its human counterpart. It thus supports the human hand's diverse techniques for grasping and handling objects. The objectives are to enhance the strength and endurance of the human hand, to extend humans' scope of action and to secure them an independent lifestyle even at an advanced age.

Since all the joints and their drive units are located outside the actual hand in the form of the exoskeleton, this manual orthosis can be fitted not only over the human hand, but also over an artificial handmade of silicone. Using the same hardware, this enables a scenario that creates a link between robotics and orthotics in a completely new way.

The ExoHand combines human intelligence with the capabilities of a robot. While machines are precise, robust and powerful, their responses to complex situations are limited. They normally rely on the visual and tactile perception and decision-making capacity of a human operator.

With the ExoHand the operator has the sensation of feeling the shape of the remote object. The human sense of touch can thus be implemented over long distances and can even be applied at the interface of the real to the virtual world. The system amplifies the strength of the human hand and helps employees remain in the work process for a longer time without suffering permanent physical effects. To prevent fatigue, the ExoHand can be worn for activities carried out in the assembly process, thereby functioning as an assistance system that makes for more pleasant working conditions in the assembly environment. The ExoHand moving by eight pneumatic actuators which ensure the precise movement of the robotic arm. The forces, the angles and distances recorded by sensors [13].

Given the fact that the construction is fully operational, but there is no literature to be found with some effort to make simulations in the MatLab Simulink, neither any experimental results of its usage. The model of this paper performs robust control and can simulate human movement using six pneumatic actuators, which in fact will ensure the accurate movement of the robotic hand in real time.

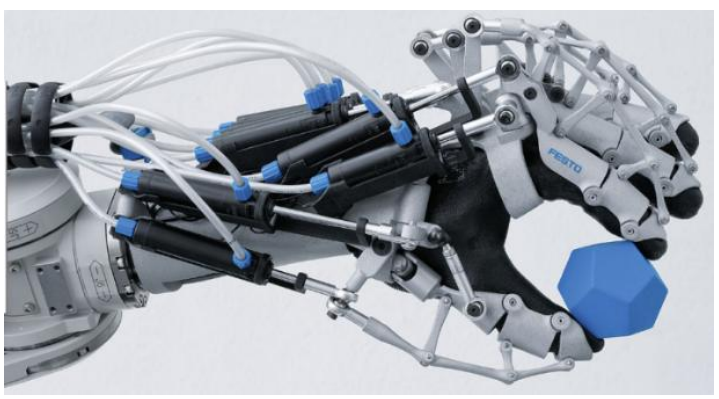

Fig 8: A Well Known Pneumatic Arm

\section{WHAT IS TO FOLLOW}

By taking advantage of the possibilities given by Matlab/Simulink the pneumatic system that was designed for the purpose of finding the most optimal method of control, could be benefit by adding fuzzy logic controller. The particular controller is not found in the literature, but could be 
used to control the position of the six pistons of the system, providing new comparison data and therefore new results. Also, checking the position of the six parallel-connected pistons could be done by placing in the system a Linear Variable Differential Transformer LVDT. In more details, Fuzzy logic is a wide scientific field created by the need to bypass the strict tradition of binary logic in which only the statement true or false exist. Fuzzy logic inserts the definition of truth degrees which measure whether an object is involved in a fuzzy set. The truth degree value is define by the membership functions which take values between 0 and 1 that describe whether an object is involved in a fuzzy set. Fuzzy control occurred from the need for a control strategy that is not based on a mathematical model system, which usually differs from the real.

The controllers designed based on fuzzy logic have the capacity to handle fuzzy data and noise data. They are based on the human ability to perceive the behavior of qualitative rules system. Through a series of simple linguistic rules, fuzzy logic can model the knowledge and experience of an experienced user. That way a knowledge based system is formed to guide basic models to be more receptive of the human logic. That way fuzzy logic control succeeds where other classic methods of control can't.

The main structural elements of a fuzzy logic controller are the following:

- Knowledge base in which the rules are stored to control the process (if-then rules)

- Fuzzy sets, which are used to represent the input and output variables of the verbal terms.

- Fuzzifier which converts the real input values in fuzzy sets

- Inference engine, which processes the outputs of the Fuzzifier and uses the knowledge base to extract the fuzzy sets and the conclusions

- Defuzzifier, which converts the conclusions into real terms, so that it can be transferred into control action of the process.

There are various types of fuzzy controllers in which the main difference lies in the inference mechanism. Essentially, entailment wherein the inference mechanism is based on statements and the type of the controller. The main types of controllers are:

- Mamdani, defined as: "If $\mathrm{x}$ is $\mathrm{A}$ then $\mathrm{y}$ is B" was named after Ebrahim Mamdani, who was among the $1^{\text {st }}$ to establish the fuzzy The outputs of the rules of this method are fuzzy sets.

- Sugeno - Takagi, which is defined as: "If $\mathrm{x}$ is A then $y$ is c", where $c$ is a number or a crisp fuzzy set.

- Takagi - Sugeno - Kang or T-S-K, is an extension of the previous rule and is one of the most important rules who is used in advanced fuzzy systems "If $\mathrm{x}$ is A then $\mathrm{y}$ is $\mathrm{c} 0+\mathrm{c} 1 \mathrm{x}$ ", where $\mathrm{c} 0, \mathrm{c} 1 \in \mathrm{R}$ [11].

Another future challenge is to undertake the simulation of a Linear Variable Differential Transformer - LVDT is actually a sensor for the linear displacement. The range of the measured displacement varies from $0,1 \mathrm{~mm}$ to $1000 \mathrm{~mm}$. Linear variable differential transformers are instruments with precision and have low divergence $( \pm 0.5 \%)$, because their core is not in contact with the coils, resulting in a very low mechanical friction. Despite their advantages LVDT are instruments used for measuring small displacements, but they are quite sensitive and are affected by vibrations and temperature. The device comprises three coils, two secondary and a primary one, the centre of the primary is a core made of soft magnetic material (soft iron, amorphous wire, FeSiB etc.).

The primary is powered with high frequency voltage and the secondaries are connected in series with opposite polarity. When the magnetic core is located in the centre, due to symmetry of the voltages induced in the secondary coils are equal and opposite connected, the output is zero. Movement of the core generates a signal (alternating voltage) in the output amplitude is proportional to the displacement and the phase indicates the direction of motion.

The name of a specific sensor is described in the working principle:

1. The adapter is subjected to the electromagnetic induction principles.

2. It has one primary and two secondary coils, which are connected and provide the difference in the trends in their respective outputs, for this reason it's called differential.

3. The magnetic coupling between the coils can be changed by affecting the magnitude of the induced electromotive force, for this reason it is considered variable.

4. LVDT is designed in a specific way, so that the changes of the magnetic coupling between the coils can be linear.

The function for the primary is given below:

$$
\begin{gathered}
V_{r e f}=V 1 \cdot \eta \mu(\omega t) \\
V=V 1-V 2=V_{0} \cdot \eta \mu(\omega t+\varphi)
\end{gathered}
$$

The LVDT finds multiple applications in the industrial department because of his many benefits:

- To control the water level in a tank.

- In engineering workshops.

- In robotics.

○ In force, pressure and acceleration measurement systems.

- For position control in hydraulic and pneumatic rams.

- For crane control [12].

The LVDT can be used for the successful control of the position of one or more pneumatic actuators of a system, providing proper control and reliable results.

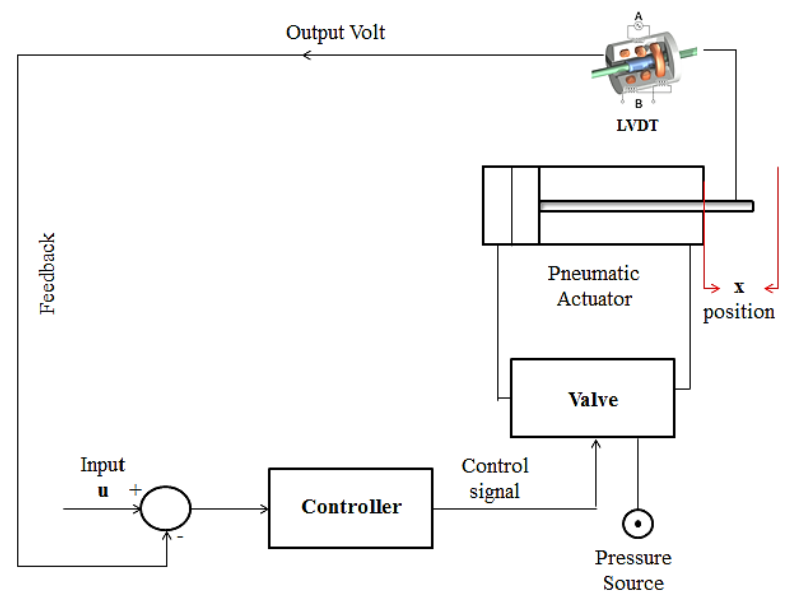

Fig 8: LVDT system with single-rod 


\section{ACKNOWLEDGMENTS}

All authors would like to express their gratitude to the PostGraduate Program of Studies "Automation of Productions and Services" of PUAS, for the financial support to undertake this research project

\section{REFERENCES}

[1] Hydraulic-Pneumatic systems and Applications, Athanasios T. Routoulas, Contemporary Publications, Athens, Greece, 2008.

[2] Thesis: Movement control in the pneumatic systems, N. Themelis, Department of Mechanical Engineering, National Technical University of Athens, Greece, 2007.

[3] Information paper: Introduction to Pneumatic Systems, I. Lignos, P. Bouslis, G. Politis, G. Chamilothoris, Electrical Sector, Region Training Center, Ioannina, Greece, 2001.

[4] Automation and Automatic Control Systems, Part A, I. Lignos, P. Bouslis, G. Politis, G. Chamilothoris, Electrical Sector, Athens, 2001.

[5] Paper \#: A High Performance Pneumatic Force Actuator System Part 1 - Nonlinear Mathematical Model, Edmond Richer and Yildirim Hurmuzlu, Southern Methodist University, School of Engineering and Applied Science, Mechanical Engineering Department, Dallas, 2001.

[6] Paper \#: Mathematical And Simulink Model Of The Pneumatic System With Bridging Of The Dual Action Cylinder Chambers, Vladislav Blagojević, Miodrag
Stojiljković, Faculty of Mechanical Engineering Niš, University of Niš, Vol. 5, No 1, 2007.

[7] Thesis: "A comparative Investigation Into ProportionalIntegral-Derivative Control Methods for Accurate Position Control of Linear Pneumatic Actuators", M.G. Papoutsidakis, University of the West of England, Bristol, UK, 2004.

[8] Paper \#: An Analysis of a Pneumatic Servo System and Its Applications to a Computer-Controlled Robot, S. Liu and J. E. Bobrow, Journal of Dynamic Systems, Measurement and Control, vol 110, PP 228-235, 1988.

[9] Paper \#: Non-conventional adaptive control of a servopneumatic unit for vertical load positioning, C. Ferraresi, P. Giraudo, G. Quaglia, West Technical Conference, 1994.

[10] Paper \#: Variable Structure Control of a Pneumatic Actuator, Tang J. and Walker G., Transactions of the ASME, vol.117, pp.88-92, 1995.

[11] Thesis: Control wind turbine induction motor squirrel cage using fuzzy logic, C. Kokkotas, Department of Electrical and Computer Engineering, University of Patras, Greece, 20013.

[12] Lab Notes: Sensors-Interfaces, C. Tsonos, Department of Electronics Engineering, University of Applied Sciences, Central Greece, 2011.

[13] New scope for interaction between humans and machines https://www.festo.com/net/SupportPortal/Files/156734/B rosch_FC_ExoHand_EN_lo.pdf 